Int. J. Gynaecol. Obstet., $1982 \quad 20: 327-334$

International Federation of Gynaecology \& Obstetrics

\title{
MULTIPLE PRIMARY NEOPLASMS OF THE OVARY AND UTERUS
}

\author{
YEW CHEONG CHOO ${ }^{a}$ and BERNARD NAYLOR ${ }^{b}$ \\ Division of Gynecologic Oncology, Department of Obstetrics and Gynecology ${ }^{\mathbf{a}}$, and Department of Pathology ${ }^{\mathbf{b}}$, University of \\ Michigan Hospital, Ann Arbor, MI, USA \\ (Received September 21st, 1981) \\ (Accepted February 2nd, 1982)
}

\begin{abstract}
Choo YC, Naylor B (Division of Gynecologic Oncology, Department of Obstetrics and Gynecology, and Department of Pathology, University of Michigan Hospital, Ann Arbor, MI, USA). Multiple primary neoplasms of the ovary and uterus.

Int J Gynaecol Obstet 20: 327-334, 1982

Multiple primary neoplasms arising in the ovary and uterus were analyzed in 55 patients: 49 synchronous and 6, metachronous. When they occurred synchronously, $74.5 \%$ of the ovarian carcinomas and $93.6 \%$ of the uterine carcinomas were stage I lesions. The endometrial carcinomas were invariably well differ. entiated and superficial. It was the stage of the ovarian carcinomas that determined the prognosis of these patients. Ways of their identification as separate neoplasms are discussed. The potential to develop further neoplasms in the gastrointestinal tract and breasts should be borne in mind.
\end{abstract}

Key words: Multiple neoplasms; Ovary; Uterus; Ovarian carcinoma; Endometrial carcinoma

\section{Introduction}

Multiple neoplasms in the ovary and uterus may arise as a result of estrogenic influence from a functioning ovarian tumor
[1], or when a hormonally active stroma is present in an epithelial ovarian carcinoma [2]. In addition, despite their distinct and separate development, both the ovaries and uterus receive contribution from the celomic epithelium which forms the ovarian germinal epithelium as well as the endometrium. Such an embryologically-derived field, when subjected to a common carcinogen, may also give rise to multiple neoplasms [3]. The identification of neoplasms in the ovary and uterus, as being separate, is clearcut when the histology is dissimilar, when the his tology is identical, no definite criteria exist, however, in their distinction as to whether they are independent or metastatic neoplasms. Although such entities are not a rarity, reports on multiple neoplasms of the ovary and uterus are scarce [3-5]. Also, it is not uncommon to encounter patients diagnosed as having ovarian carcinomas metastatic to the uterus or vice versa who have survived many years, suggesting that perhaps these neoplasms are of different origin. We undertook a retrospective study on patients with multiple neoplasms in the ovary and uterus encountered during a 27.5-year period with a view to review their clinical and pathological features and to establish guidelines for their identification.

\section{Materials and methods}

The data were obtained from the Tumor 
Registry of the University of Michigan Hospital on all patients who had multiple neoplasms of the ovary and uterus diagnosed between January 1943 through June 1980. The patients' records were analyzed with respect to their presentation, diagnosis, treatment and follow-up. The pathology slides were reviewed and the findings correlated with that at surgery. Fifty-nine patients were identified; five were excluded after analysis because actual evidence of separate neoplasms could not be established. Thus synchronous neoplasms in the ovary and uterus were found in 49 patients and metachronous neoplasms in six patients. Although the study embraced such a long period, most patients were identified within the past decade. In order to establish criteria for their identification as separate origin, observations were focused on the following: (1) operative findings; (2) morphology of the two neoplasms; (3) the extent of endometrial neoplasm and its association with hyperplasia, atypia and carcinoma in situ; (5) transition of the ovarian carcinoma from germinal epithelium; and (6) the stroma of the ovary.

\section{Results}

\section{A. Synchronous multiprimary neoplasms of the ovary and uterus}

The patients' ages ranged from 27 years to 81 years, with a mean of 54.6. Their parity ranged from 0 to 7 , the average being 2.1 Fourteen patients $(28.6 \%)$ were nulliparous, and $31(63.0 \%)$ were postmenopausal. Obesity, diabetes and hypertension were not significant findings as was the family history of cancer. Exogenous estrogen was recorded in eight patients.

The most common symptom was abnormal uterine bleeding, noted in 20 patients $(40.8 \%)$, while abdominal discomfort, bloating and distension were the complaints in 13 patients $(26.5 \%)$. Only eight patients (16.3\%) had both uterine bleeding and abdominal symptoms. Three patients presented with acute abdomen and three patients were completely asymptomatic. The most common finding was an enlarged uterus, recorded in 14 patients $(28.6 \%)$. Thirteen patients $(26.5 \%)$ had an ovarian tumor palpable and separate from a normal sized uterus. Findings of dual pathology of an enlarged uterus and an ovarian mass were noted in only three patients.

The pre-treatment diagnoses are shown in Table I. Twenty-four patients were diagnosed as having endometrial carcinoma following a D \& C; of those, seven (14.3\%) had a diagnosis of ovarian tumor as well because of a palpable ovarian mass. In ten patients, the sole diagnosis was ovarian neoplasm. In ten other patients, the diagnosis varied and included fibroid, carcinoma of the colon, torsion of the ovary, acute appendicities and diverticular abscess.

At surgery, 45 patients were found to have a uterus that was mobile and grossly uninvolved by the ovarian neoplasm. On the basis of dissimilar histology, 22 patients were diagnosed as having two neoplasms arising in the ovary and uterus. Figures 1 and 2 illustrate such an example. In the remaining 27 patients with neoplasms of identical histology, 25 has endometrioid carcinoma of the ovary associated with adenocarcinoma of the uterus. Recognition of variable patterns and grades led to further categorization of the neoplasms as separate in 12 of these patients (Figs. 3 and 4). In nine patients, a primary adenocarcinoma arising in the uterus was evident by the presence of associated atypia and carcinoma in situ, or by its origin in endometrial polyps.

Table I. Synchronous ovarian and uterine neoplasms, pre-treatment diagnosis.

Uterine carcinoma

17

Uterine carcinoma and ovarian mass $\quad 7$

Ovarian neoplasm $\quad 10$

Pelvic mass, undetermined 5

Miscellaneous

10

TOTAL 49 


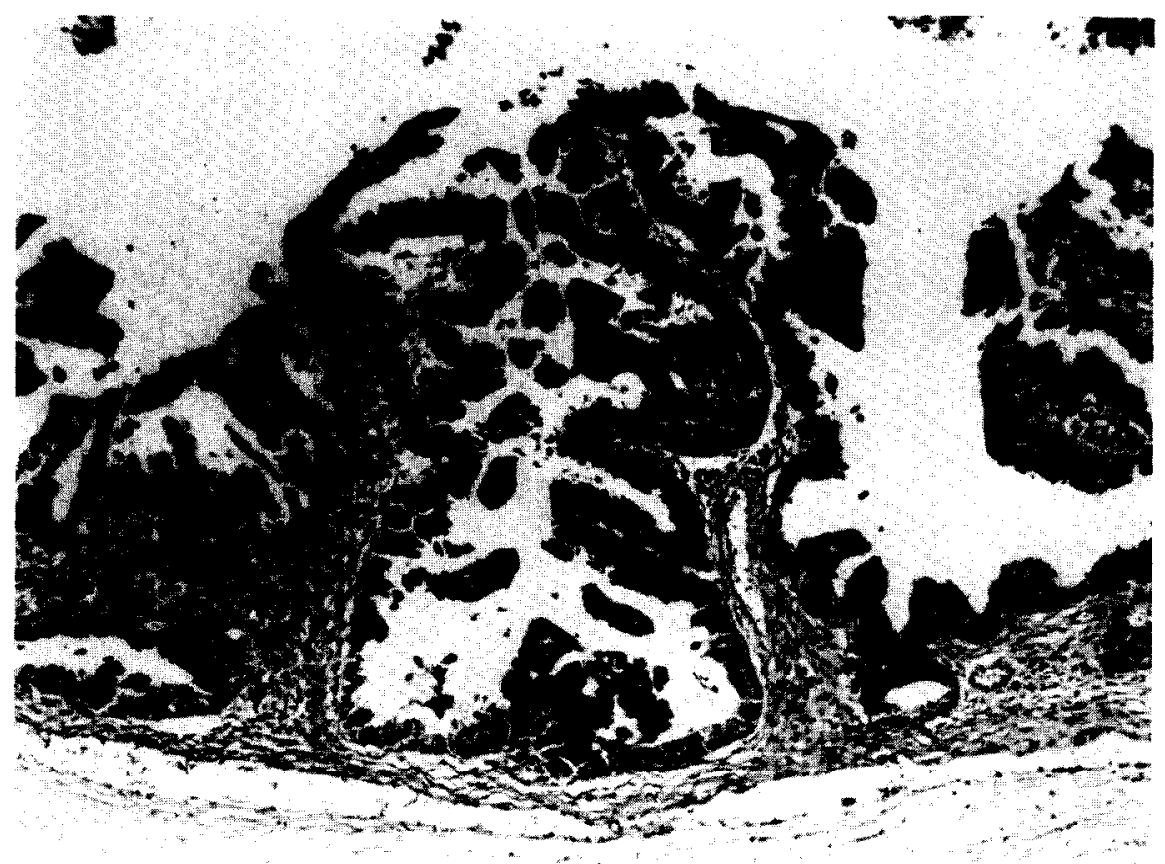

Fig. 1. Ovary. Moderately differentiated papillary serous cystadenocarcinoma of ovarian origin $(\mathrm{H} \& \mathrm{E}, \mathrm{X83})$.

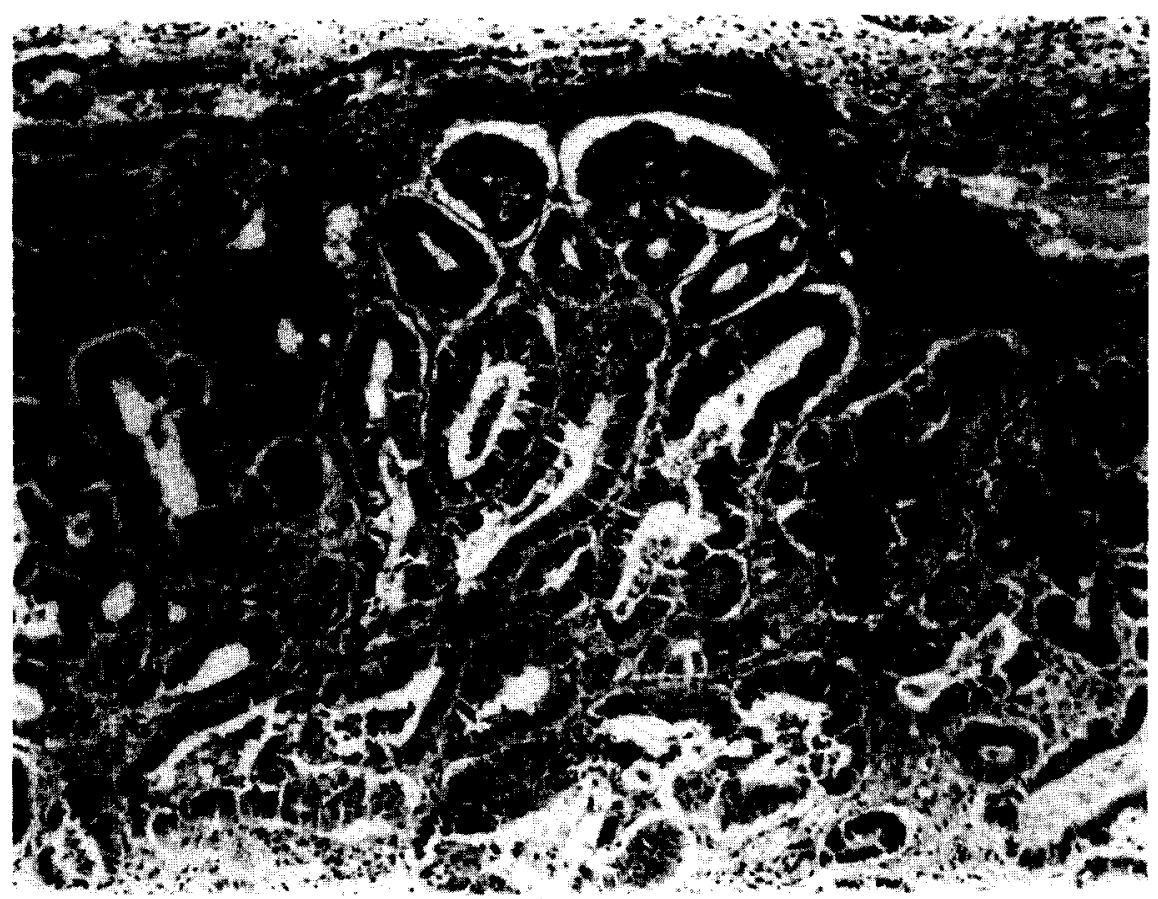

Fig. 2. Endometrium Moderately differentiated adenocarcinoma which originated in endometrium. It is morphologically distinct from the ovarian lesion (H \& E, X83). 


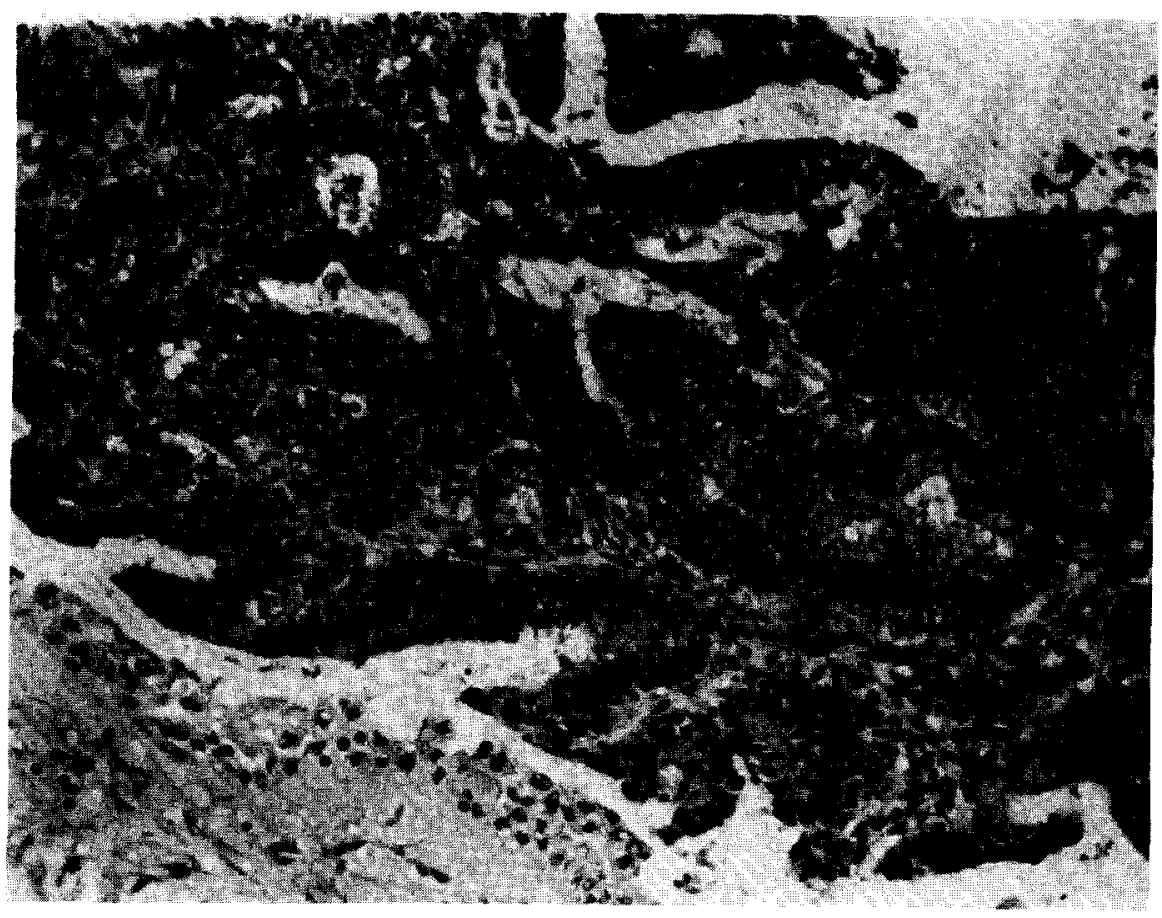

Fig. 3. Ovary. Moderately differentiated adenocarcinoma of ovarian origin (H \& E, $\times 132$ ).

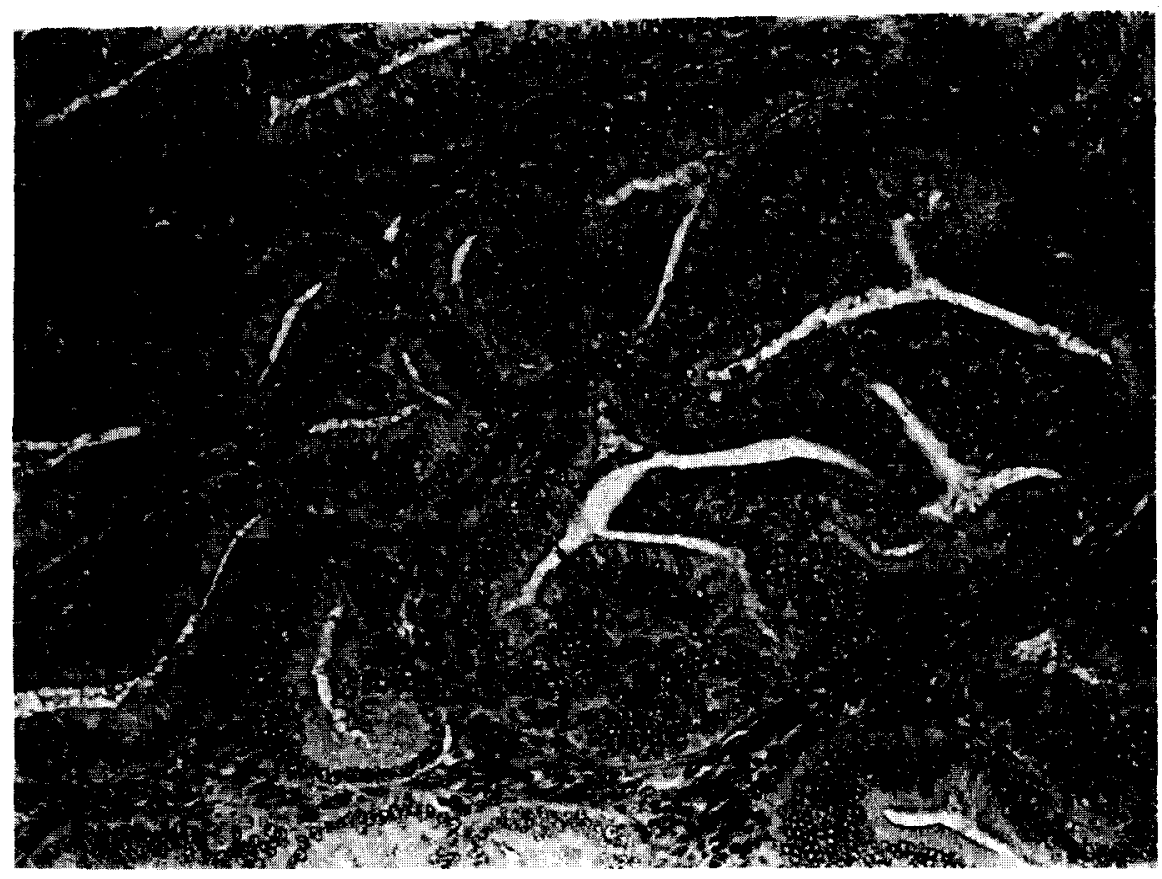

Fig. 4. Endometrium. Well differentiated adenocarcinoma of endometrial origin (H \& $\mathrm{E}, \mathrm{X} 132)$ 
Six patients with endometrioid carcinoma of the ovary had evidence of malignant transformation from endometriosis or transition from an inclusion cyst and cystadenoma.

The histology of the uterine neoplasms is shown in Table II. Forty-seven were adenocarcinomas and two were leiomyosarcoma. The endometrial carcinomas were well differentiated in 37 patients $(78.8 \%)$, they were focal, confined to the endometrium or invading less than one-third of the endometrium in 32 patients $(68.1 \%)$.

The most common ovarian carcinoma was endometrioid, identified in 25 patients $(51.0 \%)$, followed by serous cystadenocarcinoma in 16 patients $(32.6 \%)$. Bilateral ovarian carcinomas, most commonly serous variety, occurred in 14 patients $(28.6 \%)$ (Table III). Stromal hyperplasia in the neoplastic or normal ovary was noted in five patients (10.2\%) (Fig. 5).

When the uterine and ovarian carcinomas were independently staged, it was found that 44 patients $(93.6 \%)$ had stage I endometrial carcinomas, of which $32(68.1 \%)$ were IAG1 lesions, and 35 patients $(74.5 \%)$ had stage I carcinomas of the ovary.

The treatment modalities although varied, were conventional. Surgery and postoperative irradiation were the most common treatment employed (24 patients), and 11 patients received adjunctive chemotherapy as well.

Table 2. Synchronous ovarian and uterine neoplasms, histology of uterine neoplasms.

\begin{tabular}{llllc}
\hline & $\begin{array}{l}\text { Well } \\
\text { differ- } \\
\text { entiated }\end{array}$ & $\begin{array}{l}\text { Moderately } \\
\text { differ- } \\
\text { entiated }\end{array}$ & $\begin{array}{l}\text { Poorly } \\
\text { differ- } \\
\text { entiated }\end{array}$ & Total \\
\hline $\begin{array}{c}\text { Adeno- } \\
\text { carcinoma }\end{array}$ & 32 & 7 & 1 & 40 \\
$\begin{array}{c}\text { Acantho- } \\
\text { adeno Ca }\end{array}$ & 5 & 1 & 1 & 7 \\
$\begin{array}{c}\text { Leiomyo- } \\
\text { sarcoma }\end{array}$ & 37 & 8 & 2 & 49 \\
\hline \begin{tabular}{c} 
TOTAL \\
\hline
\end{tabular}
\end{tabular}

Table 3. Synchronous ovarian and uterine neoplasms, histology of ovarian neoplasms.

\begin{tabular}{lrrr}
\hline & Unilateral & Bilateral & Total \\
\hline Endometrioid Ca & 21 & 4 & 25 \\
Serous cystadeno Ca & 9 & 7 & 16 \\
Mucinous cystadeno Ca & 2 & 0 & 2 \\
Clear cell Ca & 1 & 1 & 2 \\
Mixed epithelial Ca & 2 & 2 & 4 \\
TOTAL & 35 & 14 & 49 \\
\hline
\end{tabular}

Overall, 42 patients were available for 3and 5-year survival analysis. The 3- and 5 -year corrected survivals were $83.3 \%$ and $73.8 \%$, respectively. Among the 33 patients with stage I ovarian and uterine carcinoma, the corresponding 3- and 5-year corrected survivals were $87.5 \%$ and $78.1 \%$, respectively.

\section{B. Metachronous multiprimary neoplasms of the ovary and uterus}

Sequential multiprimary neoplasms were found in six patients. In five patients, the initial neoplasm was ovarian and a second carcinoma arose in the retained uterus after a mean interval of 6.5 years; four of them had been given postoperative pelvic irradiation as well. A hormonal factor may also be operative in two of them as one patient had a malignant thecoma and the other received stilbesterol for a prolonged duration. The uterine carcinomas in all these patients were stage $I$, and at hystcrectomy none had demonstrable residual ovarian neoplasm. Three of the patients have died of intercurrent disease and the other two are alive at 3 years and 7 years, respectively. In the six th patient, the initial carcinoma was uterine, and 14 years later, a stage IV ovarian carcinoma was found. Significantly, her initial uterine carcinoma was treated with irradiation only.

Of the entire series of 55 patients with synchronous and metachronous neoplasms of the ovary and uterus, seven $(12.7 \%)$ have developed additional neoplasms elsewhere for a total of ten carcinomas (Table IV). The most common site was the colon followed by 


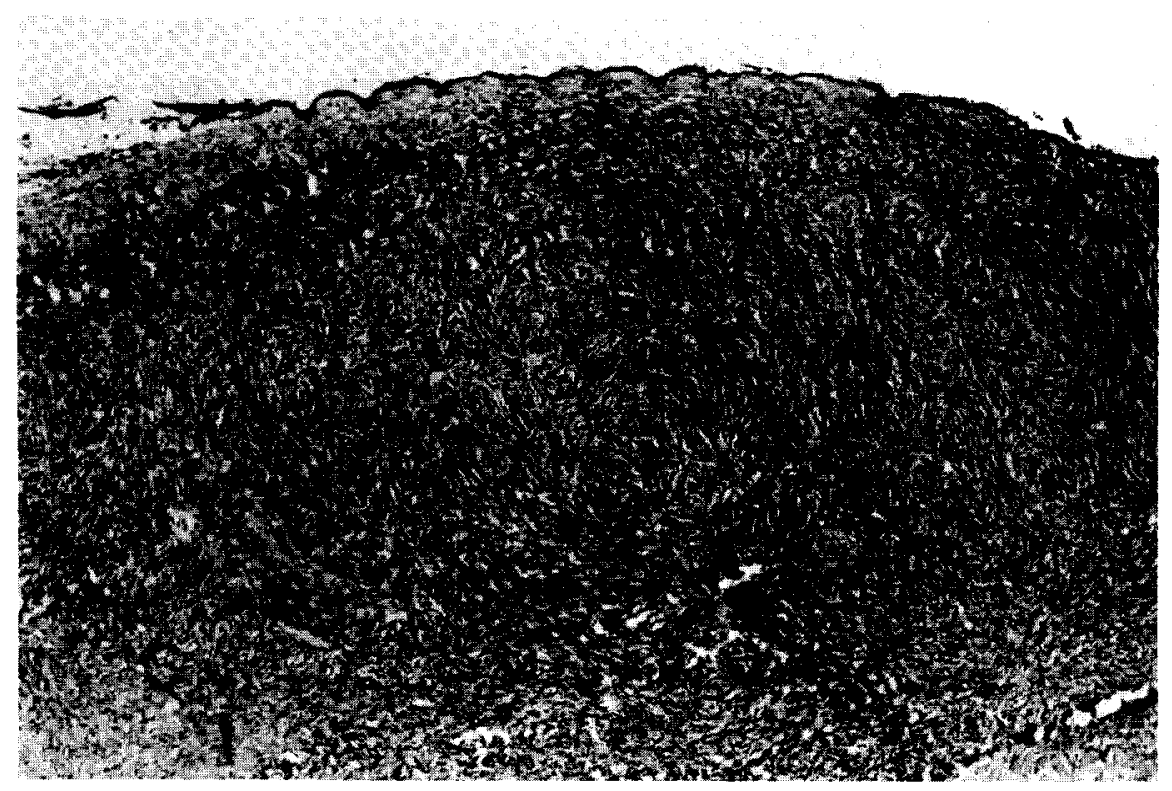

Fig. 5. Ovary. Cortical stromal hyperplasia (H \& E, X53).

the breast. It is noteworthy that in one patient, initial simultaneous carcinomas arising in the right colon, ovary and uterus were found, and 7 years and 10 years later, she developed a fourth and fifth carcinoma in the stomach and sigmoid colon, all successfully treated.

\section{Discussion}

Neoplasms arising in the ovary and uterus are not always associated with the presence of a functioning ovarian tumor. Only one malignant thecoma was identified in our series while the rest of the ovarian neoplasms

Table 4. Synchronous and metachronous ovarian and uterine neoplasms, additional neoplasms in seven patients.

\begin{tabular}{ll}
\hline Colon $\mathrm{Ca}$ & 3 \\
Breast $\mathrm{Ca}$ & 2 \\
Stomach $\mathrm{Ca}$ & 1 \\
Jejunum, Ca & 1 \\
Appendix, Ca & 1 \\
Cervix, Ca & 1 \\
Bladder $\mathrm{Ca}$ & 1 \\
\hline
\end{tabular}

were epithelial in origin. Epithelial tumors, however, may be estrogenic when stromal hyperplasia is present. This was found in only $10.2 \%$ of our patients. Rome [6] reported a higher incidence of stromal hyperplasia in epithelial carcinomas arising in postmenopausal ovaries. Such a phenomenon is said to be a response to the mechanical presence of the epithelial carcinoma [2]. Woodruff [3] postulated that multiple malignancies of the ovary and uterus may arise in a commonlyderived epithelium due to carcinogenic influence. This may well happen in patients who develop bilateral ovarian carcinomas simultaneously with endometrial carcinomas. It should also be realized that carcinogenesis from radiation treatment is a possibility as this could not be discounted in five of our six patients who developed a metachronous carcinoma in the retained uterus or ovary.

It should be noted that symptoms and signs suggesting dual neoplasms in the ovary and uterus are often absent and their diagnosis are seldom made preoperatively. Such a suspicion should be raised when a patient 
is found to have endometrial carcinoma and an ovarian mass is also palpable. It is also significant that 21 of our patients $(42.9 \%)$ had no bleeding whatsoever, this perhaps is attributable to an early endometrial carcinoma. It is therefore mandatory in an occasional young patient who has ovarian carcinoma and in whom conservation of the uterus may be a consideration, that a diagnostic $D \& C$ be performed to exclude an occult cndometrial carcinoma. In most other situations, the treatment for an ovarian malignancy should be the extirpation of the ovaries and uterus lest a carcinoma should develop in the retained uterus.

When an ovarian tumor is found to occur in association with endometrial carcinoma, an exploratory laparotomy should be initiated. Following surgery, adjuvant irradiation and/or chemotherapy may then be instituted.

The categorization of their origin when the ovarian and uterine carcinomas are of similar histology is not at all simple; this is especially true of endometrioid carcinoma of the ovary associated with an adenocarcinoma of the uterus. Kottmeier [7] reported that onethird of all patients with endometrioid carcinoma of the ovary had concomitant adenocarcinoma of the endometrium. At surgery, one should therefore clearly define whether the uterus and ovaries are free of contiguous spread by neoplasm. Endometrial carcinoma that arises de novo is suggested by finding hyperplasia, atypia and carcinoma in situ as well. The endometrial component in such cases is invariably well differentiated, focal and superficial. It is well known that the fallopian tube may serve as a conduit for the spread of a carcinoma from the uterus to the ovary [8]. The ovary may then be secondarily involved by extracapsular implants but there would be no transition of the surface epithelium. Endometriosis is commonly found in association with endometriod carcinoma of the ovary, and one should look for its malignant transformation. Noting for disparity between the grade and pattern of the two neoplasms requires careful pathological evaluation, and when present, it is evidence to regard both neoplasms as having separate origins. When both neoplasms are anaplastic, however, such a distinction is not always possible, and one has to regard the neoplasms as metastatic especially when deep myometrial invasion is also evident. The excellent survival of these patients is further evidence that the neoplasms are of independent origin, for if the neoplasms are metastatic, the prognosis should be correspondingly poor. One should also note that since the uterine carcinomas are often well differentiated and superficial, it is the stage of the ovarian carcinomas that ultimately determines the prognosis and survival of these patients.

Our study, as well as others [9-11], has shown that multiple primary neoplasms may not only develop in the ovary and uterus but they also commonly occur in association with carcinomas in the gastrointestinal tract and breast. Such an awareness is essential in the evaluation and follow-up of these individuals.

\section{References}

1 Gusberg SB, Kardon P: Proliferative endometrial response to theca-granulosa cell tumor. Am J Obstet Gynecol 111: $635,1971$.

2 Janovski DA, Paramanandhan TL: Enzymatically active stromal cells in ovarian tumors. In Ovarian tumors, Chap. 9, p 119. WB Saunders Co. Philadelphia, London, Toronto, 1973.

3 Woodruff JD, Julian DG: Multiple malignancy in the upper genital canal. Am J Obstet Gynecol 103: 810, 1969.

4 Campbell JS, Magner D, Fournier P: Adenoacanthoma of ovary and uterus occurring as co-existent or sequential primary neoplasms. Cancer 14: 817, 1961.

5 Silverman BB, O'Neill RT, Mikuta JJ: Multiple malignant tumors associated with primary carcinoma of the ovary. Surg Gynecol Obstet 134: 244, 1972.

6 Rome RM, Laverty CC, Brown JB: Ovarian tumours in postmenopausal women. $\mathrm{J}$ Obstet Gynaecol $\mathrm{Br}$ Commonw 80: 984, 1972.

7 Kottmeier HL: The diagnosis and treatment of ovarian malignancies. Arq Patol 37: 51, 1965.

8 Anbrokh GB, Anbrokh M: Mechanism of development and morphology of secondary carcinomas of the oviducts 
in primary uterine corpus carcinoma. Neoplasma 23: $549,1976$.

9 Schoenberg BS, Greenberg RA, Eisenberg H: Occurrence of certain multiple primary cancers in females. J Natl Cancer Inst 43: 15, 1969.

10 Schottenfield $\mathrm{D}$, Berg $\mathrm{J}$ : Incidence of multiple primary cancers. IV. Cancer of the female breast and genital organs. J Natl Cancer Inst 46: 161, 1971.

11 Moertel CG: Multiple primary malignant neoplasms. Historical aspects. Cancer 40 1786, 1977.
Address for reprints

Dr. Y.C. Choo

Department of Obstetrics and Gynecology Queen Mary Hospital

University of Hong Kong

Hong Kong 